Doug Geisler, Eva K. Grebel, and Dante Minniti, eds.

\title{
The Outer Halo Cluster System of NGC 1399
}

\author{
B. Dirsch, D. Geisler, T. Richtler \\ Universidad de Concepción, Chile \\ J.C. Forte \\ Universidad Nacional de La Plata, Argentina
}

\begin{abstract}
.
We investigate the outer halo globular cluster population of NGC 1399. This study uses wide-field imaging of this cluster system, which covers the largest area studied with CCD photometry until now. The cluster system of NGC 1399 is found to extend further than $100 \mathrm{kpc}$ from the galaxy. A population of metal-rich, as well as metal-poor clusters has been identified at these large radii. At radii smaller than $55 \mathrm{kpc}$ the specific frequency of the red cluster system remains constant, while that of the blue clusters increases proportional to $r^{0.8 \pm 0.2}$. For larger radii, the uncertainty of the galaxy light profile does not permit any reliable statement.
\end{abstract}

\section{Introduction}

NGC 1399, the central galaxy of the Fornax cluster, is, besides M87, the nearest central $\mathrm{cD}$ galaxy in a relatively dense cluster. Hanes \& Harris (1986) already noted in an earlier photographic work that the cluster system extends further than $13^{\prime}$, which corresponds to a distance of $70 \mathrm{kpc}$ at the distance of NGC 1399 (assumed to be $19 \mathrm{Mpc}$ ). While the properties of the inner cluster system are well known (Ostrov et al. 1998), the population structure of the outer cluster system was virtually unknown. New wide-field CCD cameras make studies of this extended system now feasible.

\section{Observations \& Reduction}

MOSAIC wide-field images $\left(36^{\prime} \times 36^{\prime}\right)$ in the Washington $C$ and the Kron-Cousins $R$ filter have been obtained at the CTIO $4 \mathrm{~m}$ telescope centered near NGC 1399 (in Dec. 1999). In addition a background field 3.5 degrees north-east of NGC 1399 has been observed. We used SExtractor and DAOPHOT for identification and photometry of the objects, respectively. Later spectroscopic observations with the VLT showed that the success rate for the identification of GCs between $2^{\prime}$ and $8^{\prime}$ based on this photometry was higher than 95\% (Richtler et al. 2001). In total, we identified about 5000 cluster candidates out to a radial distance of $22^{\prime}$ and down to a limiting magnitude of $\mathrm{R}=24$. 


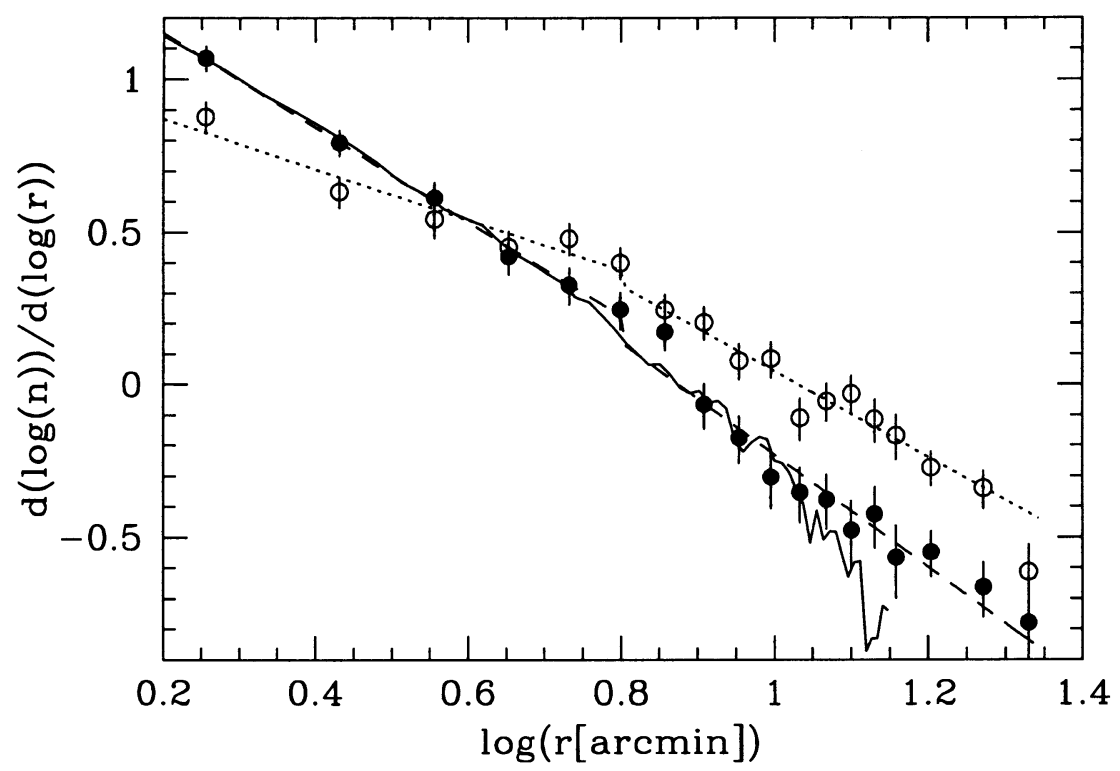

Figure 1. The radial density profiles of red (filled circles) and blue (open circles) globular clusters are plotted together with the fitted power-laws. The solid line shows the galaxy light profile in the $\mathrm{R}$ filter.

\section{The different cluster populations}

For the current study we selected only clusters brighter than $R=23$, well above the completeness limit. The globular cluster candidates were divided into blue clusters $(1<C-R<1.4)$ and red clusters $(1.5<C-R<2.2)$. The reason for doing that is the strong color bimodality of the cluster system. The color difference of $C-R=0.47$ between the peak values of these two populations is most probably due to a mean metallicity difference of approximately 1 dex. Bimodal distributions are typical for large cluster galaxies as shown by Gebhardt $\&$ Kissler-Patig (1999) and Forbes \& Forte (2001). The mean metallicity values for the two populations are $[\mathrm{Me} / \mathrm{H}]=-0.3 \pm 0.15$ and $[\mathrm{Me} / \mathrm{H}]=-1.3 \pm 0.15$.

The two populations have rather different spatial distributions with the blue clusters being less concentrated than the redder ones, as shown in Fig.1. Moreover, each population changes its power-law index at about $7^{\prime}$. We find surface density profiles for the blue population proportional to $r^{-0.8 \pm 0.15}$ and $r^{-1.4 \pm 0.14}$ (for the inner and outer part, respectively), and for the red population profiles proportional to $r^{-1.5 \pm 0.15}$ and $r^{-1.8 \pm 0.18}$.

The galaxy light profile measured by us in the $R$ band closely matches the radial distribution of the red cluster population within $11^{\prime}$. Outside of $11^{\prime}$ small background uncertainties make a definite statement on the surface brightness profile impossible. The blue cluster system on the other hand has within $11^{\prime}$ a shallower profile and therefore its specific frequency with respect to the $\mathrm{R}$ band luminosity increases outwards proportional to $r^{0.8 \pm 0.2}$. 
The $B$ band surface brightness profile determined by Caon et al. (1994) and our profile are in excellent agreement, which indicates that no color gradient in $B-R$ is visible within $11^{\prime}$. Together with the fact that the red clusters closely follow the galaxy light, the question remains why the dominance of the blue clusters at larger radii is apparently not accompanied by a color gradient in the galaxy light. However, more accurate photometry at low surface brightness levels is required to resolve this issue.

To investigate the very remote cluster population, we selected all cluster candidates which are further away than $10^{\prime}$ from any bright Fornax galaxy and more than $13^{\prime}$ away from NGC 1399. The blue peak of the color distribution of these remote clusters has the same color and the same width as the blue peak of the inner blue clusters. A red peak on the other hand cannot unambiguously be identified; however, a shoulder at the position of the red peak of the inner population can be seen. We estimate the mean metallicity of this red cluster population to be roughly -0.6 dex. The similarity of the color distribution of the remote blue clusters suggests that they are still associated with the outer halo of NGC 1399, indicating that its cluster system extends to beyond $100 \mathrm{kpc}$. An analogous statement for the red clusters is more difficult.

It remains an intriguing question whether these clusters and the "intergalactic" clusters observed by Bassino et al. (2001) are connected. They found a population of clusters at large distances from the center from the Fornax cluster that have a very similar metallicity distribution as our remote cluster sample. One might speculate that this population is a tracer of a global stellar population spread out over the Fornax cluster.

\section{Acknowledgments}

We acknowledge the help of M. Shara, D. Zurek and E. Grebel, who obtained some images that were used in this investigation.

\section{References}

Bassino L., Forte J.C., 2002, IAU Symp. 207, this volume

Caon N., Capaccioli M., D'onofrio M., 1994, A\&AS 106, 199

Forbes D.A., Forte J.C., 2001, MNRAS 322, 257

Gebhardt K., Kissler-Patig M., 1999, AJ 118, 1526

Hanes D.A., Harris W.E., 1986, ApJ 309, 564

Ostrov P.G, Forte J.C., Geisler D., 1998, AJ 116, 2854

Richtler T. et al. 2002, IAU Symp. 207, this volume 